\title{
Social media and storytelling: tools to raise engagement with physics
}

\author{
Valentina Scotti ${ }^{a, b, *}$ \\ ${ }^{a}$ Dipartimento di Fisica, Università degli Studi di Napoli Federico II, \\ Complesso Universitario di Monte Sant'Angelo, Napoli, Italy \\ ${ }^{b}$ Sezione di Napoli, Istituto Nazionale di Fisica Nucleare, \\ Complesso Universitario di Monte Sant'Angelo, Napoli, Italy
}

E-mail: scottiv@na.infn.it

According to the Global Digital Report 2020, social media active users reach 51\% of the world population, with an annual growth of $10 \%$. Due to the penetration of social media in everyday life, many scientific collaborations and institutions are present on different social media platforms and recognize the role they play in reaching a wider audience. Social media engagement can positively influence not only scientific communication, but also the day-to-day job of scientists involved, since their presence on social networks can increase their networking and impact beyond the research community.

As a case study, the experience gained as a social media manager of INFN Napoli is presented. Social media were used as a tool to convey a correct message about research activities and to share the emotional side of research. In this paper, the strategies implemented on different platforms, and a way to use storytelling to increase the engagement are reported. Social media proved to be very powerful in connecting physicists with the non-specialized public, in particular with students, and each other.

Increasing the visibility and engagement of physicists can have a huge impact on how nonphysicists see scientists and their relationships to science and the scientific community.

40th International Conference on High Energy physics - ICHEP2020

July 28 - August 6, 2020

Prague, Czech Republic (virtual meeting)

\footnotetext{
${ }^{*}$ Speaker
} 


\section{Introduction}

In order to understand social media importance in science communication, it is useful to analyze the actual impact of social media on society. A useful report of data concerning the use of the internet and social platform can be found in the Global digital Report [1]. This report is published every year by Hootsuite and Wearesocial, and it summarizes the status of the digital world; besides, it includes social media trends and strategies for the following year.

According to the Global digital Report 2020, social media active users reach 51\% of the world population, with an annual growth of $10 \%$. These numbers are even more impressive considering that children under 13 years old are not officially allowed to sign up on social media.

Nowadays for the population under 55 years old, social media are the main source of news, with a peak of $66 \%$ among young people (under 24 years of age). The most popular social media platform used as a news source is Facebook, followed by YouTube. A study conducted in the USA reports that internet and social media are the primary source of science and technology information for $81 \%$ of young people [2]. The conclusion to be drawn from this overview is clear: science communication cannot avoid the use of social media.

A wide-known model for science communication is the Public Understanding of Science [3]. In this framework, the communication flows in a top-bottom uni-directional way. This model is also called the "deficit model" since it starts from the assumption that the public lacks knowledge. Thus, scientists need to learn how to communicate with the public and consider scientific communication as their duty. The underlying idea is that "The more you know, the more you love it".

In early 2000, in addition to the Public Understanding model, the Engagement model [4] became very popular. According to this model, the public is not passive and the communication flows in two ways. Since the audience plays an active role in the communication, scientists are encouraged to share as much as possible with the broader public. The Engagement model implies that outside their specialist fields, scientists are part of the public too. Public engagement is seen as necessary to obtain public confidence in science.

\section{Social media and science}

As seen in the previous section, social media play an significant part in everyday life. Moreover, social media allow scientists to get in contact with a large and diversified public, not typically approached through traditional science outreach and communication activities.

Research institutes and experiments need to be present and active on new communication channels with adequate strategies [5], not only to reach a wider audience but also to deliver a correct message and counteract mistrust [6], and to have real-time interactions and direct connections with the broad public.

Needless to say, the communication style must be appropriate. In a white paper prepared for the American Association for the Advancement of Science [8] it is recommended that, when communicating to the broad audience, scientists invert the path they usually follow to deliver their messages, starting from the main results and then going to the importance of the studies, explaining the impact of their research on society, and only finally describing the details. According to the 
white paper on "Public Engagement with and Communication of Science in a Web-2.0 Media Environment", the message delivered should be short, meaningful, and memorable.

When correctly used, social media engagement can positively influence not only scientific communication, but also the day-to-day job of scientists involved, since their presence on social networks can increase their networking and impact beyond the research community [7]. In fact, there is a positive relationship between social media news use and trust in science across different societies: social media news use is more strongly related to trust in science than traditional news use [6].

Finally, according to the Global Digital Report 2020 [1] one of the latest trend in social media marketing is the Employer Generated Content, since these type of posts are perceived as more sincere. For this reason, each member of the institution needs to be a brand ambassador and telling the stories behind the research has become fundamental. This trend highlights the importance of narratives for public engagement with science. Storytelling can capture and retain the attention of audiences, along with conveying clear messages that make science and its results relevant to people's lives [9].

Due to the penetration of social media in everyday life, also in the field of Physics many scientific collaborations and institutions are present on all the social media platforms and recognize the role they play in communicating with a broader audience [10]. Recently, there has been a shift in the kind of pictures posted on social media by Physics-related accounts. A few years ago, it was more common to see images related to detectors or data, nowadays the focus is on researchers and the spotlight is on their personal effort and story.

\section{The experience of INFN Napoli}

In the last part of this paper, the experience managing different social media platforms for the Division of Naples of the Italian Institute of Nuclear Physics (INFN) will be illustrated as a case study.

INFN is the Italian research agency dedicated to the study of the fundamental constituents of matter and the laws that govern them, under the supervision of the Ministry of Education, Universities, and Research. The INFN is fully committed to promoting scientific culture. It takes part in outreach activities in Italy and it organizes various exhibitions and events throughout the country [11]. The division of Naples employs 89 people (researchers, technicians, administrative staff), and around 300 associated researchers [12].

Social media are used by INFN Napoli to complement their outreach activities. The main aims are:

- to make their research activities known to their community;

- to engage with young people sharing young researcher stories and successes;

- to build awareness and personal branding among the employees.

The social media manager of INFN Napoli works in collaboration with the national INFN Communications Office [13]. To increase the engagement on social media of INFN Napoli, the following strategies [16] were implemented: 
- usage of emotions as a tool to connect with the public: studies in cognitive and social psychology show that human thinking is affected by emotions [14]. As a result, concepts and messages that appeal to emotions and affect tend to increase the retention of the message [15].

- construction of social media posts based on the pyramid of communication flow of the AAAS [8]: since the attention span on social media is short, it is mandatory to go straight to the main point.

- implementation of the general communication rule "Keep It Short and Simple", which becomes even more important when using social media. Since $99 \%$ of the users access social media through their smart-phones [1], it is necessary to build a communication strategy that is quick and well delivered through a small screen. The typical length of the caption showed on social media on a mobile device is between 110 and 240 characters, and these are useful numbers to keep in mind to deliver the message successfully. The constraint on the length of the text makes even more poignant the need for good pictures.

\subsection{Social media strategies}

In particular, INFN Napoli social media manager decided to target different social media platforms at different publics.

Facebook was chosen to build awareness among the researchers and the staff of the Institute, highlighting the contribution given by people working at INFN Napoli to scientific collaborations they participate in, and the impact of their work on the national and international scientific community.

INFN Napoli shares on Facebook different kinds of posts, celebrating results of their researchers and sharing the work done by their technicians, using storytelling to make the message clearer for a non-specialist audience. Looking at the insight statistics data provided by Facebook, it is noticeable the big difference in numbers of likes, comments, and sharing, when the shared post makes the followers feel involved. Figure 1 shows the success of different post types based on average reach and engagement, data clearly show that both the reach and the engagement increase when the shared content is a picture. During the last year, the Facebook page of INFN Napoli saw a 30\% increase in the number of followers, and in the reach of all the kind of contents, all the posts are now more liked, commented and shared.

While on Facebook the most shared contents are pictures and celebratory posts, to build a community sense, the strategies implemented for other social media platforms aim at reaching different audiences.

Twitter is mainly devoted to interaction with other institutions and sharing more scientific news.

Instagram turned out to be a very effective and engaging tool for young people. The average age of the followers of INFN Naples on Instagram is lower compared to the one of the followers on Facebook. Instagram is interesting also for the possibility to use stories, a type of post which only lasts for 24 hours and allows to have more interaction with followers. This type of post is particularly useful to post live videos of outreach activities and to engage young people or students in small quizzes or challenges related to the event. 


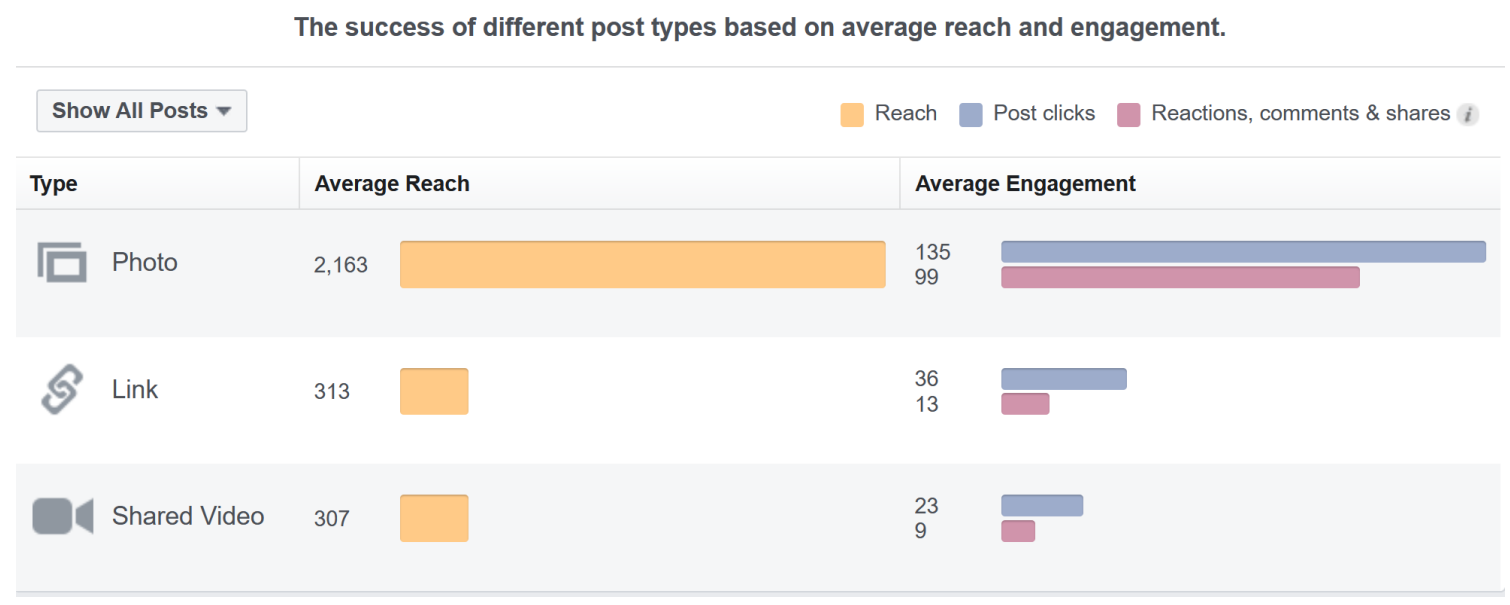

Figure 1: The success of different post types based on average reach and engagement.

Finally, INFN Napoli is present also on YouTube. This page was used chiefly as a repository for old videos since INFN Napoli could not invest more resources on it. Nonetheless, some recordings of seminars reached an unexpected number of views. YouTube is often described as a tool to reach younger people, surprisingly, the age group of INFN Napoli followers is the oldest part of the demographic (i.e., over 55 years of age), this might be related to the fact that most of the video uploaded have a long duration.

The increased impact of the social media profiles of INFN Napoli was demonstrated also by the grow of articles on local and national newspaper which shared the news. To summarize, the overall result is a positive perception of the institution and its influence beyond the research community.

\section{Conclusions}

Nowadays, social media are a necessary tool in science communication and their use allow physicist to improve public engagement with their research. Storytelling can increase the attention of audiences, helping to make researchers' results relevant to the non-specialist public.

It is possible to use different social media to get in contact with wide and diversified audiences. In the case of INFN Napoli, the sharing of positive and inspiring news proved to be a useful tool to build a community and also to reach more people outside the research institute.

\section{References}

[1] https://wearesocial.com/digital-2020

[2] https://journals.sagepub.com/doi/full/10.1177/2056305118797720

[3] Bodmer W., "The Public understanding of science", Royal Society, 1985

[4] Holden C., "From PUS to PEST", Science, Vol. 298, pp. 49, 2002

[5] https://jcom.sissa.it/sites/default/files/documents/jcom0201(2003)F01.pdf 
[6] Fostering public trust in science: The role of social media https://doi.org/10.1177/0963662519869097

[7] https://www.nature.com/articles/d41586-019-03869-7

[8] Yeo, S. K., "Public Engagement with and Communication of Science in a Web-2.0 Media Environment", The American Association for the Advancement of Science (AAAS), 2015

[9] Downs, J. S. , "Prescriptive scientific narratives for communicating usable science", Proceedings of the National Academy of Sciences, 111(Supplement 4), 13627-13633, 2014, doi: 10.1073/pnas.1317502111

[10] https://phys.org/news/2018-04-social-media-scientists-message.html

[11] https://home.infn.it/

[12] https://www.na.infn.it/

[13] Mazzotta, F.,"Physics on social media: impact and interaction to deal with fast communication,” PoS EPS-HEP2017 (2017), 561 doi:10.22323/1.314.0561

[14] Lodge, M., et Taber, C. S., "The Rationalizing Voter", Cambridge University Press, 2013

[15] Heath, C., et Heath, D., "Made to stick: Why some ideas survive and others die", Random House, 2007

[16] Telling stories in science communication: case studies of scholar-practitioner collaboration https://jcom.sissa.it/archive/18/05/JCOM_1805_2019_N01 